\title{
Molecular Dynamics of A Biglycan-Rosmarinic Acid Complex with Focal Adhesion Kinase for Possible Arrest of Metastasis in Non-Small Cell Lung Cancer (NSCLC): An In-Silico Study
}

\author{
Highland Hyacinth*1, Thakur Monica ${ }^{2}$, Pandya Pujan ${ }^{3}$, Mankad Archana ${ }^{3}$, George Linz-Buoy 1 \\ 1 Department of Zoology, Biomedical Technology and Human Genetics, School of Sciences, Gujarat University, Ahmedabad-380009, Gujarat, \\ India \\ 2 Department of Biochemistry, School of Sciences, Gujarat University, Ahmedabad-380009, Gujarat, India \\ ${ }^{3}$ Department of Botany, Bioinformatics, School of Sciences, Gujarat University, Ahmedabad-380009, Gujarat, India
}

\begin{abstract}
Background: Non-small cell lung cancer (NSCLC) is the major cause of mortality all over the world. Significant increase of biglycan is seen in the lung cancer cells when compared with the normal cells. It promotes tumor invasion and metastasis by activating Focal Adhesion Kinase (FAK) signaling pathway. The increased FAK activity may contribute to the metastatic potential of malignant tumors. This study was carried out to establish binding interactions of some selected phytocomponents against biglycan for the possible arrest of metastasis.
\end{abstract}

Methods: Protein-ligand interaction studies were performed using 30 natural compounds from different culinary herbs having potential therapeutic role against the target protein biglycan (BGN).

Molegro Virtual Docker (v 5.0) was used as docking tool to evaluate the effectiveness of selected phytocomponents based upon the interaction with the protein's active site residues with minimal binding energy. Protein-protein docking was performed to observe the interaction of BGN and FAK using Hex (v 8.0.0). Molecular dynamics (10 ns) of BGN-RA-FAK and FAK-RA-BGN was performed in Yasara structure (v 17.8.15) which showed stability of the structure in terms of RMSD values.

Results: Molecular docking analysis revealed the selectivity of Rosmarinic acid (RA) towards BGN and FAK. Molecular dynamics trajectory of BGN-RA-FAK and FAK-RA-BGN complexes showed the stability of structure in terms of Time vs Energy and Time vs RMSD values and revealed that binding of RA to BGN will block the interaction of FAK.

Conclusions: Hence, investigating the binding interactions of BGN-RA-FAK complex may turn out to be helpful in arresting metastasis in NSCLC.

Keywords: Non-small cell lung cancer, Biglycan, Focal adhesion kinase, Phytocomponents, Molecular Docking, Molecular Dynamics

Article Info: Received 12 July 2019; Review Completed 20 August 2019; Accepted 26 August 2019; Available online 30 Aug 2019

\section{Cite this article as:}

Highland H, Thakur M, Pandya P, Mankad A, George LB, Molecular Dynamics of A Biglycan-Rosmarinic Acid Complex with Focal Adhesion Kinase for Possible Arrest of Metastasis in Non-Small Cell Lung Cancer (NSCLC): An In- Silico Study, Journal of Drug Delivery and Therapeutics. 2019; 9(4-A):159-166 http://dx.doi.org/10.22270/jddt.v9i4-A.3382

\section{*Address for Correspondence:}

Highland Hyacinth, Department of Zoology, Biomedical Technology and Human Genetics, School of Sciences, Gujarat University, Ahmedabad380009, Gujarat, India

\section{INTRODUCTION}

Lung cancer is the leading cause of cancer related deaths and non-small cell lung cancer (NSCLC) is the most frequent subtype comprising of $85 \%$ of recorded cases ${ }^{1}$. According to the cancer facts and figures ${ }^{2}$ of American cancer society, the five year survival rate for lung cancer is only $18 \%$. It constitutes about 6.9 per cent of all new cancer cases and 9.3 percent of all cancer related deaths in both sexes in India 3 and continues to be a major global health problem. The disease is diagnosed in more than 1.6 million new patients each year. However, significant progress is ongoing in both the prevention and treatment of lung cancer 4. Many integrated multidisciplinary approaches to the treatment of lung cancer have improved patient outcomes, but there is an urgent need for a novel therapeutic strategy for lung cancer as there is no breakthrough treatment with long-term efficacy ${ }^{5}$. Specifically improved proteomics techniques for the identification, detection, and verification of biomarkers 
have improved our understanding of lung cancer but, early detection of lung cancer is important for a better outcome and to decrease the lung cancer mortality rate 6,7 .

Metastasis, the spread of cancer through lymph and blood, to distant organs is the main reason of death for most of the patients with malignancy ${ }^{8}$. Hence, this study was aimed at employing an in silico approach to identify a specific binding interaction of Protein-Ligand-Protein for arresting metastasis in NSCLC.

Biglycan (BGN) is a member of small leucine-rich proteoglycans family, and it has been ascribed a pivotal role in oncogenesis and development of various other types of human cancer. It is also an important component of the extracellular matrix ${ }^{9}$. BGN is found in almost every organ in a non-uniform distribution pattern within human body. Recent studies have indicated that there is significant increase of BGN in tumor tissues as compared to normal tissues, in several cancer types. It is known as a multivalent proteoglycan which promotes tumor invasion and metastasis by activating the Focal Adhesion Kinase (FAK) signaling pathway. The increased FAK activity may contribute to the metastatic potential of malignant tumors 10 . Hence, this makes biglycan a potential target protein for the present in silico study aimed at halting metastasis.

In this study, molecular docking, Molecular dynamics and protein-protein interaction studies were employed to establish binding interactions of some selected phytocomponents against the target protein biglycan.
In the present study, Molegro Virtual Docker (MVD) v 5.0 (www.molegro.com) was used as a docking tool, along with this Molegro Molecular Viewer (MMV) was used for calculating docking score 11 . MVD software requires the receptor and ligand coordinates which is either in Mol2 or PDB format. Protein- Protein docking was done with the help of Hex (version 8.0.0) software. Protein- ligand docking and Protein- Protein docking is a very useful approach to find the binding interaction of target protein with single or multiple ligands. For molecular dynamics study YASARA Structure (version 17.8.15) was the software of choice 12 . AMBER14 force field ${ }^{13}$ was used for Molecular dynamic simulations ${ }^{14}$.

\section{Protein and Ligand Preparation:}

The X-ray crystal structures of BGN (PDB ID: 2FT3) ${ }^{15}$ and FAK (PDB ID: 4NY0) 16 were obtained from the Research Collaboratory for Structural Bioinformatics (RCSB) (http://www.pdb.org/) protein data bank (PDB) 17 having Uniprot ID P21810 and Q05397 respectively (Figure 1) and prepared for molecular docking simulation by removing all the heteroatoms (i.e., nonreceptor atoms such as water, ions, etc.). The crystallographic structures of these proteins were selected according to the maximum matched sequences among the protein chains available in RCSB PDB database. 3D structures of all the 30 natural compounds from different culinary herbs having potential therapeutic role were used as ligands for this study (Figure 2) were obtained from PubChem database (https://pubchem.ncbi.nlm.nih.gov/) 18 in 3D structure data format (SDF).

\section{MATERIALS AND METHODS}

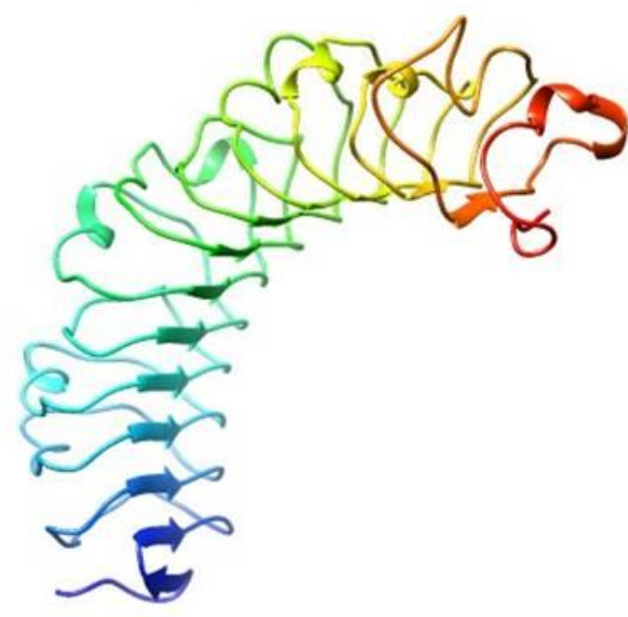

Biglycan (PDB ID: 2FT3)

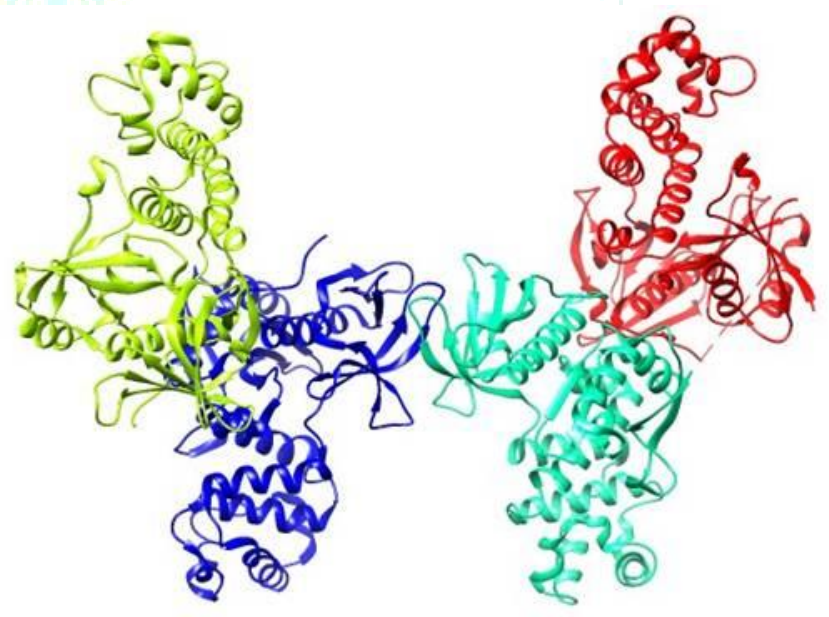

Focal Adhesion Kinase (PDB ID: 4NY0)

Figure 1: 3D structures of Biglycan (BGN) and Focal adhesion kinase (FAK) 


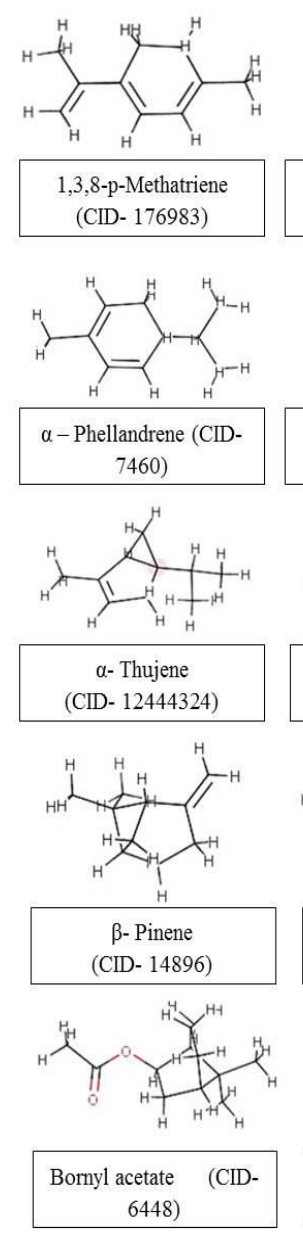

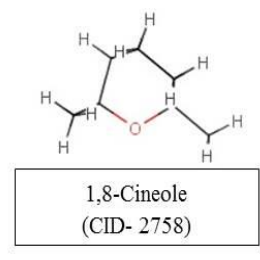

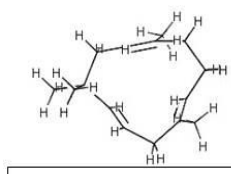

$\alpha$-Humulene (CID- 5281520)
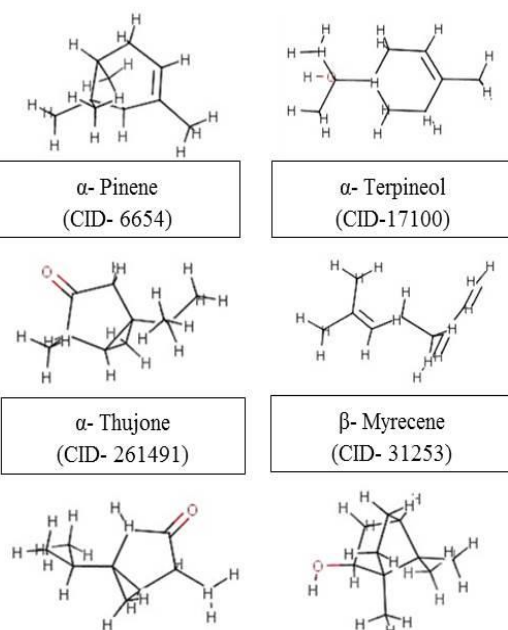

$\alpha$ - Terpineol (CID-17100)
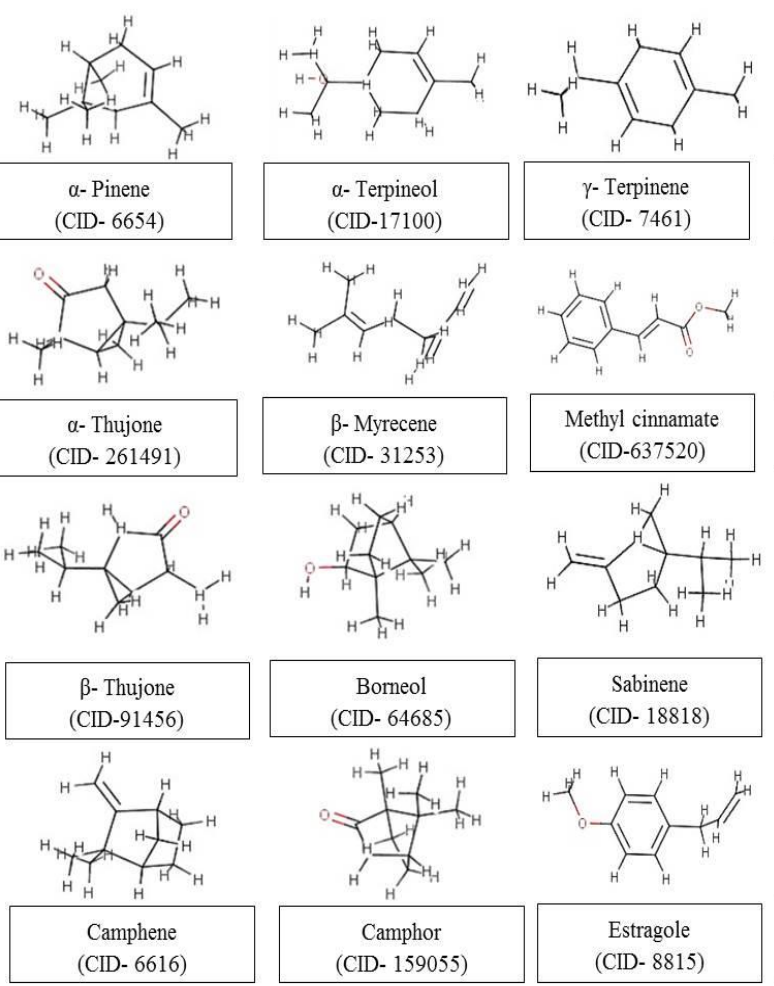

$\gamma$-Terpinene (CID- 7461)

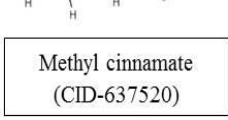
(CID-637520)
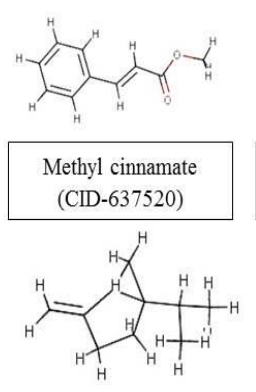
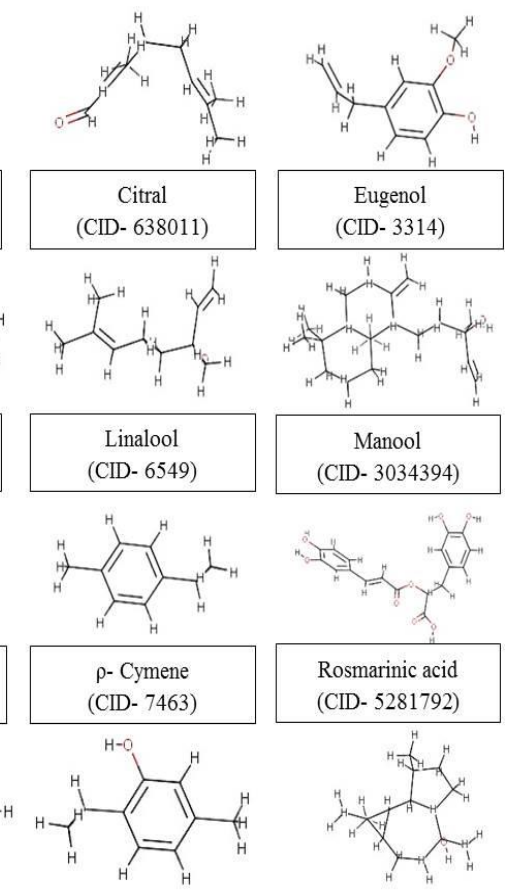

Manool (CID- 3034394)
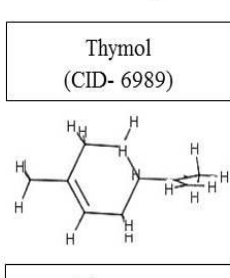

Limonene (CID- 440917)

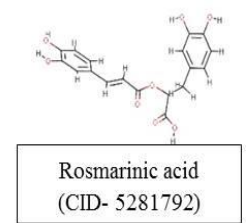

(CID- 5281792)

Figure 2: 3D structures of the selected phytocomponents from different culinary herbs

\section{Molecular Docking studies:}

\section{(A) Biglycan (BGN) and Phytocomponents (Ligands):}

Molecular docking technique was employed to dock the selected phytocomponents against BGN using Molegro Virtual Docker (MVD) to locate the interaction between the phytocomponents and the target protein BGN. All the 30 phytocomponents were docked against the target protein BGN and the compounds having minimum energy were selected for further study. Default settings were used for all the calculations.

\section{(B) FAK and selected phytocomponents (Ligands):}

The selected 30 phytocomponents from different culinary herbs were again docked against the targeted protein FAK to check the binding interaction of the phytocomponents with the protein.

\section{(C) BGN and FAK (Protein- Protein) Docking:}

Protein- Protein docking of BGN and FAK was done using Hex (version 8.0.0) protein docking software. 500 model structures were formed according to the interaction energy and bonding of the two proteins. The highest docking score was used for the further study.

\section{(D) Molecular docking of BGN-Phytocomponent complex with FAK and FAK-Phytocomponent complex with BGN:}

BGN-Phytocomponent complex and FAK-Phytocomponent complex were docked with FAK and BGN respectively to see the interaction energy of protein-ligand-protein complex which will be used further in the study.

\section{(E) Molecular dynamics simulations:}

The molecular dynamics simulation study was carried out to identify the changes in the structural conformation, interaction pattern and binding stability of BGNPhytocomponent-FAK and FAK-Phytocomponent-BGN complexes. The complex was simulated with frame capture at 10ns (Production Period) intervals to analyze the molecular dynamics (MD) trajectory.

The default simulation parameters of YASARA Structure were used for the study with fixed pressure $\mathrm{P}$, temperature $\mathrm{T}$ and number of atoms $\mathrm{N}$ (constant-NPT ensemble) with TIP3P (Three-site Transferrable Intermolecular Potential) 19 water model and amber 14 force field 20 .

The $\mathrm{pH}$ of the simulation system by default is set as the physiological $\mathrm{pH}$ of 7.4. Furthermore the MD simulation was performed at temperature $298 \mathrm{~K}, 1$ bar pressure with ion concentration as a mass fraction of $0.9 \% \mathrm{NaCl}$, with solvent density of 0.997 and at time steps of $1 \mathrm{fs}$ with the periodic boundaries wherein all the atoms present were in the mobile state. The molecular dynamics study was done to identify the stability and structural changes of the complexes in terms of time vs energy and time vs root mean squared deviation (RMSD) values 21 .

\section{RESULTS:}

Using different in-silico tools like MVD, YASARA Structure (version 17.8.15) and Hex (version 8.0.0), the results obtained revealed a beneficial specific binding interaction between the target protein and phytocomponents which may be able to arrest metastasis by inhibiting the role of biglycan in the activation of FAK. 


\section{Molecular Docking analysis:}

\section{(A) BGN and Phytocomponents:}

The ligands show binding affinity representing various types of interactions including hydrogen bond interactions, steric interactions and the responsible amino acid residues for protein-ligand interactions were His 235, Asp 237, Arg 257 Leu 258, Gly 259, Gly 261, His 262, Glu 281, His 283, Asp
285. After conformation of these key amino acid residues with all the phytocomponents, Rosmarinic acid (RA) shows the highest docking score with biglycan (Table 1). The binding poses of Biglycan and phytocomponents (Figure 3) shows that there are 5 hydrogen bond interactions with RA and the key amino acid residues were His 235, Leu 258, His 262, Glu 281 and Asp 285.
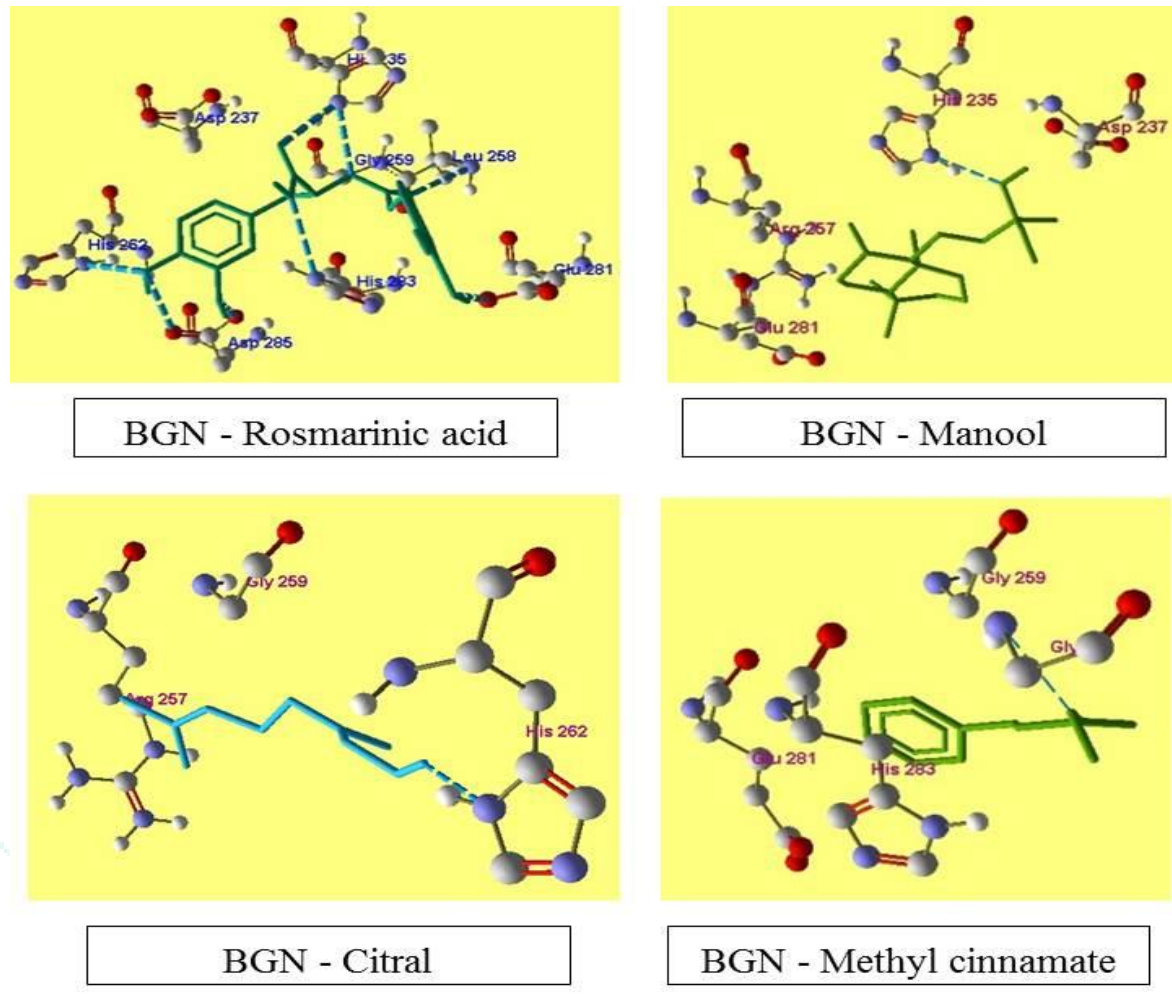

BGN - Methyl cinnamate

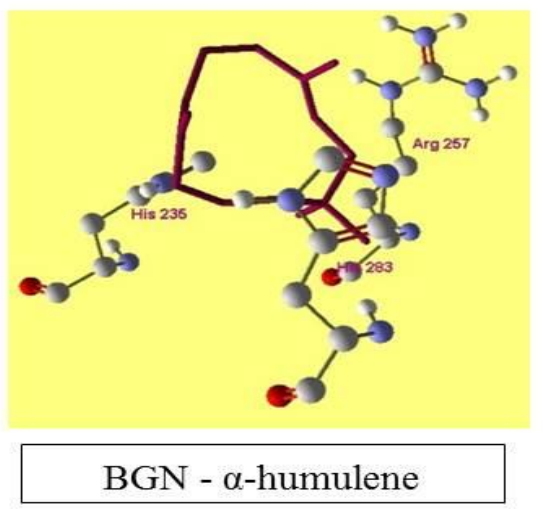

Figure 3: Binding poses of phytocomponents (Ligands) with target protein Biglycan (BGN)

Table-1: Interaction profile of Biglycan (BGN) with phytocomponents.

\begin{tabular}{|c|c|c|c|}
\hline Protein + Ligands & $\begin{array}{c}\text { Docking Score } \\
\text { (Kcal/mol) }\end{array}$ & H Bond & Steric Interactions \\
\hline BGN -Rosmarinic acid & -113.445 & $\begin{array}{c}\text { His 235, Leu 258, His 262, } \\
\text { Glu 281, Asp 285 }\end{array}$ & $\begin{array}{c}\text { His 235, Asp 237, Leu 258, Gly 259, His } \\
\text { 262, Glu 281,His 283, Asp 285 }\end{array}$ \\
\hline BGN - Manool & -88.125 & His 235 & His 235, Asp 237, Arg 257, Glu 281 \\
\hline BGN - Citral & -85.118 & His 262 & Arg 257, Glu 259, His 262 \\
\hline BGN - Methyl cinnamate & -80.631 & Gly 261 & Gly 259, Gly 261, Glu 281, His 283, \\
\hline BGN - $\alpha$-humulene & -80.254 & Nil & His 235, Arg 257, His 283 \\
\hline BGN - $\beta$-myrecene & -78.601 & Nil & His 237, Gly 259, Glu 281 \\
\hline BGN - Viridiflorol & -78.012 & Nil & Leu 258, Glu 281, His 283 28 \\
\hline BGN - Linalool & -76.605 & His 283 & Leu 258, Glu 281, His 283 \\
\hline BGN - Carvacrol & -69.675 & Leu 258, His 283 & His 235, Arg 257, Gly 259, Glu 281 \\
\hline BGN - $\alpha-$-Thujone & -64.804 & Arg 257 &
\end{tabular}




\section{(B) FAK and Phytocomponents:}

The Molecular docking study shows that FAK is effectively interacting with some of the phytocomponents and the key amino acid residues were Leu 262, Gly 263, Ser 264, Ser 265,
Trp 266, Ile 268, Glu 325, Pro 326, Leu 327 and Thr 328. Rosmarinic acid (RA) shows the highest docking score in this docking study also (Table 2). The key amino acid residues like Ser 264, Ser 265, Trp 266 and Thr 328 indicated strong hydrogen bond interaction with the protein FAK (Figure 4).
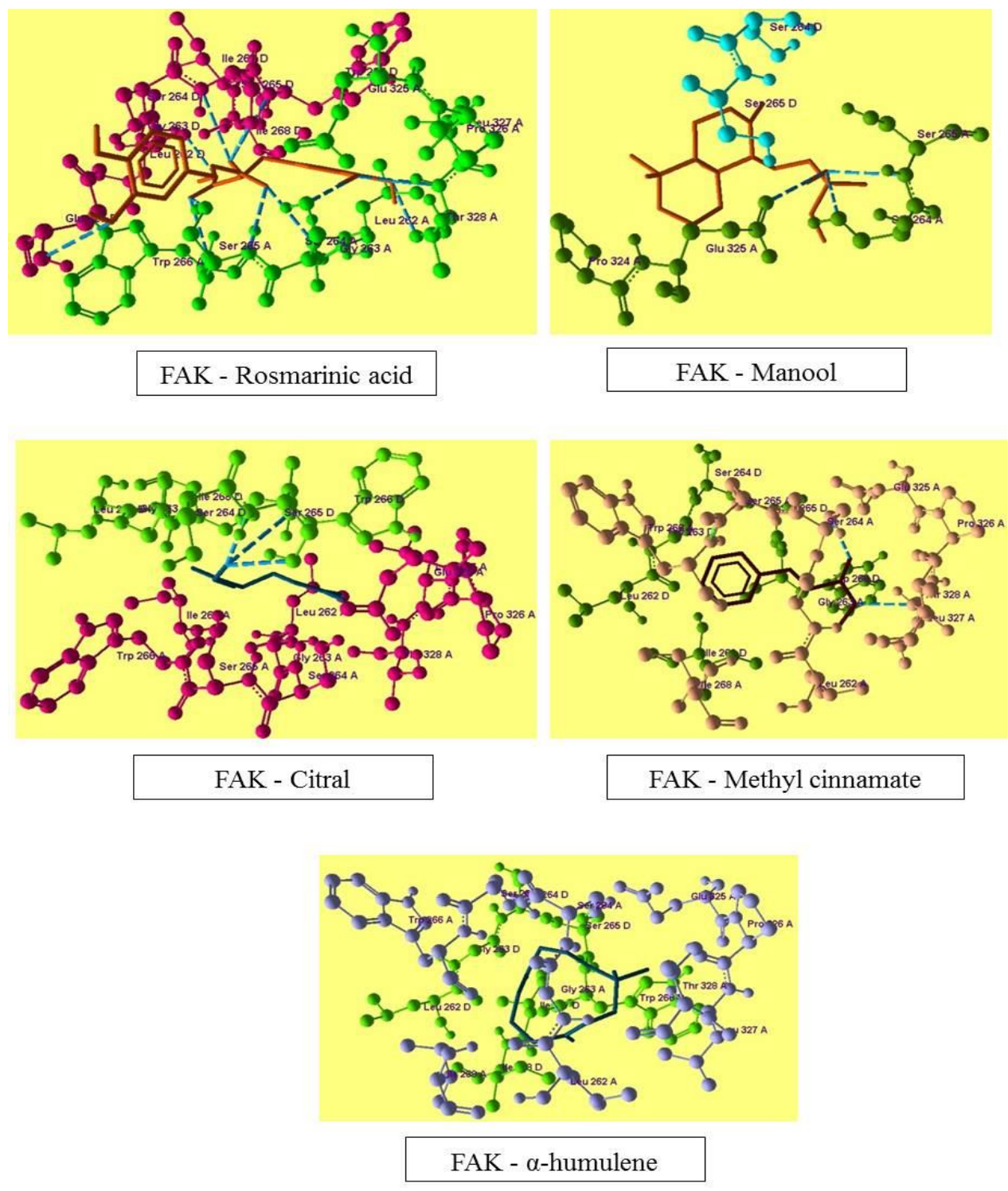

Figure 4: Binding poses of phytocomponents (Ligands) with Focal adhesion kinase protein (FAK)

Table-2: Interaction profile of Focal Adhesion Kinase (FAK) with phytocomponents

\begin{tabular}{|c|c|c|c|}
\hline Protein - Ligands & $\begin{array}{c}\text { Docking Score } \\
\text { (Kcal/mol) }\end{array}$ & H Bond & Steric Interactions \\
\hline $\begin{array}{c}\text { FAK - Rosmarinic } \\
\text { acid }\end{array}$ & -100.771 & $\begin{array}{c}\text { Ser 264, Ser 265, Trp } \\
\text { 266, Thr 328 }\end{array}$ & $\begin{array}{c}\text { Leu 262, Gly 263, Ser 264, Ser 265, Thr 328, Leu } \\
\text { 327, Pro 326, Trp 266 }\end{array}$ \\
\hline FAK - Manool & -64.236 & Glu 325 & Ser 264, Ser 265, Glu 325 \\
\hline FAK - Citral & -98.026 & NIL & Gly, Ser 265, Leu 327 \\
\hline $\begin{array}{c}\text { FAK - Methyl } \\
\text { cinnamate }\end{array}$ & -101.138 & Ser 264, Thr 328 & Gly 263, Ser 264, Ser 265, Trp 266, Leu 327, Thr 328 \\
\hline FAK - $\alpha$-humulene & -71.782 & NIL & Leu 262, Gly 263, Ser 264, Trp 266, Glu 325, Pro 326 \\
\hline FAK - $\beta$-myrecene & -91.419 & NIL & Gly 263, Ser 264, Trp 266, Leu 327, \\
\hline FAK - Viridiflorol & -78.138 & Ser 264, Ser 265, Trp & 266, \\
\hline FAK - Linalool & -98.327 & Ser 265, Trp 266 & Gly 263, Ser 264, Ser 265, Trp 266, Pro 326, Leu 327 \\
\hline FAK - Carvacrol & -91.076 & Ser 264 & Ser 264, Ser 265, Leu 327 \\
\hline FAK - $\alpha-$-Thujone & -73.190 & Ser 264 & Gly 263, Ser 264, Ser 265, Trp 266 \\
\hline
\end{tabular}




\section{(C) Protein- Protein docking results:}

To examine the binding interaction of biglycan and focal adhesion kinase, protein-protein docking was done using Hex (version 8.0.0) protein docking software. The result shows 500 model structures according to the interaction energy. The model which showed the highest binding interaction of-817.00 KJ/mol (Figure 5) was selected and used for further study.

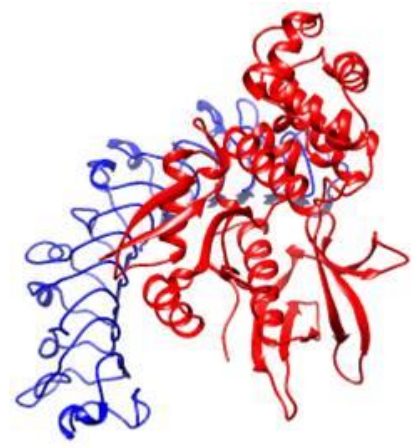

Figure 5: 3D structure of BGN-FAK complex

\section{(D) BGN-RA complex with FAK and FAK-RA complex} with BGN:

The Biglycan-Rosmarinic acid (BGN-RA) complex which was formed by the protein-ligand docking studies was then docked with FAK. The result shows that BGN-RA complex have the binding score of $-763.82 \mathrm{KJ} / \mathrm{mol}$ with the protein FAK. Similarly FAK-RA complex was docked with BGN and the interaction score noted was $-815.61 \mathrm{KJ} / \mathrm{mol}$.

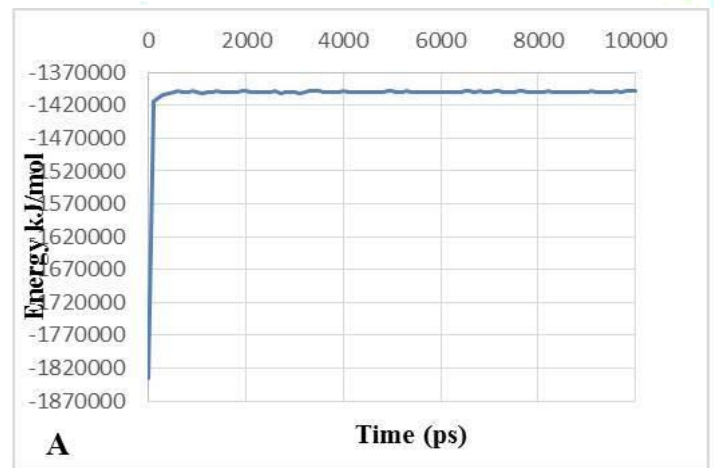

Time (ps) vs Energy (kJ/mol)

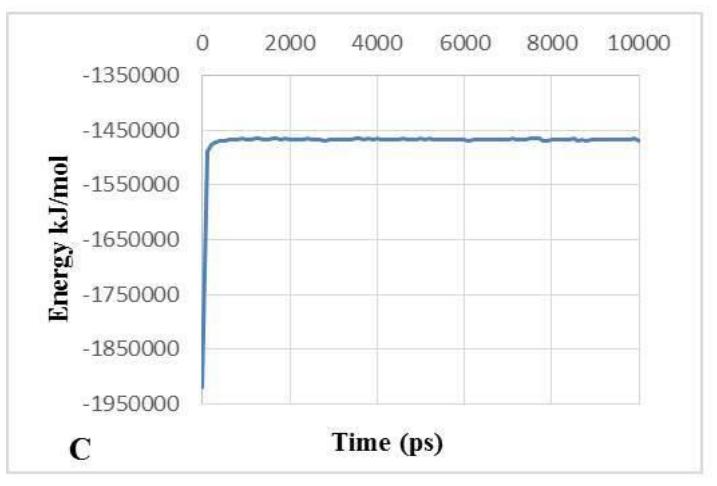

Time (ps) vs Energy (kJ/mol)

\section{Molecular Dynamics (MD) studies:}

The binding poses of BGN-RA complex with FAK and FAK-RA complex with BGN were selected for the molecular dynamics study, the stability of this complex structure was analyzed with the help of molecular dynamics study using YASARA software. The study was aimed at identifying the stability of the structure which was used for this study and to observe the initial and subsequent structural changes in the complex. The results obtained indicated stability and structural changes in terms of RMSD values. Figure 6(A) and Figure 6(C) shows the MD trajectories of the complexes in Time (ps) vs Energy (kJ/ mol) having minimum -1835908.36 and maximum -1396981.43 with an average of -1403930.128 $\mathrm{kJ} / \mathrm{mol}$ energy value for BGN-RA-FAK and minimum 1918312.348, maximum -1465364.668 and an average of $1472015.331 \mathrm{~kJ} / \mathrm{mol}$ for FAK-RA-BGN. Time (ps) vs RMSD values can also be seen in Figure 6(B) and Figure 6(D) having major peaks at $8200 \mathrm{ps}, 8300 \mathrm{ps}, 9700 \mathrm{ps}$ and $10000 \mathrm{ps}$ for BGN-RA-FAK complex and at 7700ps, 8600ps, 9400ps, $9600 \mathrm{ps}$ and $10000 \mathrm{ps}$ for FAK-RA-BGN. Structural neighborliness relied on crystallographic protein data which was utilized to identify interfacial amino acids in the protein which play an important role in the binding interaction and could eventually aid in controlling metastasis. Molecular Dynamics (MD) simulations and interaction energy analysis confirmed the selectivity of compound as potential inhibitor based on the conformational and dynamic differences observed between the residues in the Protein active sites.
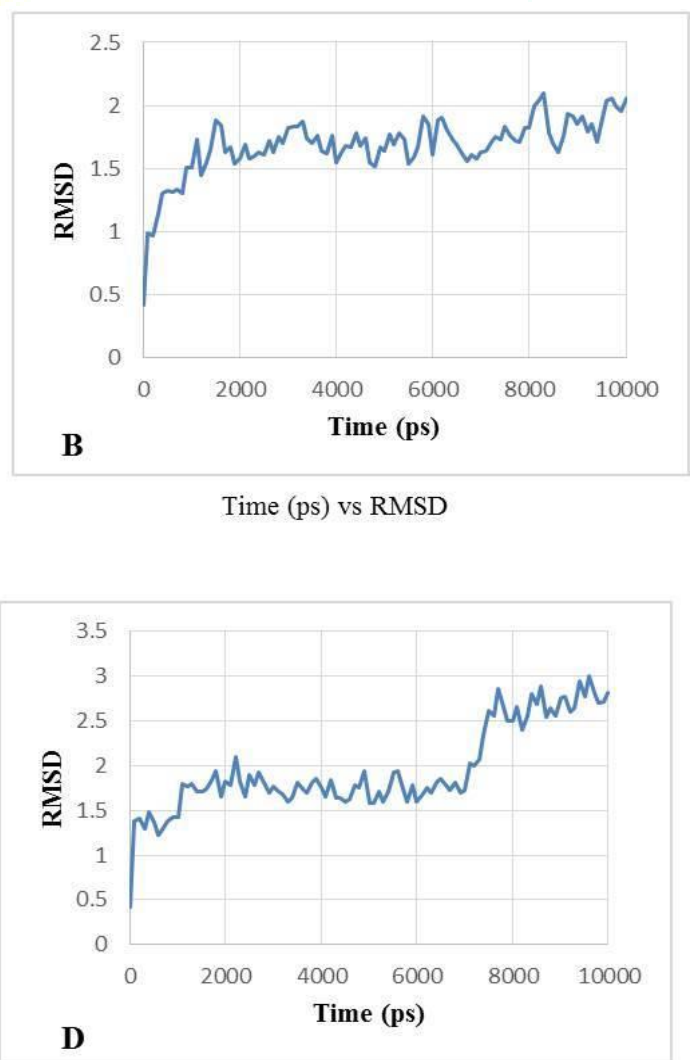

Time (ps) vs RMSD

Figure 6: A: MD Trajectories of BGN-RA-FAK complex showing Time (ps) vs Energy (kJ/ mol) B: MD Trajectories of BGN-RA-FAK complex showing Time (ps) vs RMSD C: MD Trajectories of FAK-RA-BGN complex showing Time (ps) vs Energy (kJ/ mol) D: MD Trajectories of FAK-RA-BGN complex showing Time (ps) vs RMSD 


\section{DISCUSSION:}

Metastasis is one of the major causes of mortality in patients affected with lung cancer. One of the important factors of metastasis is the orientation and composition of matrix proteins. High amounts of some of these proteins favor resistance for tumor cell migration, while some other proteins and organized oriented depositions can promote tumor cell migration. Conversely, direct cell contacts between tumor cells and fibroblasts can also create a migratory-inhibitory matrix which is composed of unorganized collagens and proteoglycans 22. Recently, Maishi and Hida 23 observed that in highly metastatic tumors, endothelial cells interact with tumor cells by secretion of a small leucine-rich repeat proteoglycan known as biglycan. Biglycan from tumor endothelial cells stimulates the tumor cells to metastasize by activating the FAK signaling pathway. FAK protein in tumors is therefore associated with increased rates of migration and invasion 24 , 25. An in-depth study by Megison et al. ${ }^{26}$, suggested that the inhibition of FAK resulted in decreased cellular migration and invasion in neuroblastoma cell lines with decreased metastasis in a murine model. Zhao and Guan ${ }^{27}$ have also explained that inhibition of FAK activity also regulates motility and cell adhesion by imparting extracellular matrix (ECM) signals from integrins to the intracellular compartment.

In the present study, selected phytocomponents from different culinary herbs manifested effective binding with Biglycan and FAK which seems to be potent in arresting the metastasis by inhibiting the role of the target protein Biglycan in the activation of FAK. These findings suggest that the selected phytocomponents could prove useful in therapeutics as blockers of key molecules that trigger metastasis. It is well known that medicinal plants are used in many countries as an alternative to synthetic drugs. Scientists are currently working towards identifying herbal extracts which could act as an active agent, to overcome world health issues 28 . These medicinal plants are rich source of phytochemicals and secondary metabolites which have been used in traditional medicine and as chemical entities for modern drugs. The medicinal value of the plant depends upon the presence of specific phytochemical components which bring about particular physiological effects in the human body. Research carried out by Ozlem et al., ${ }^{29}$ shows that the herb rosemary can inhibit the initiation and tumor progression stages in mice models. Hence in the present study, certain medicinal herbs like rosemary, sage, parsley, thyme and basil were selected, based on their potential medicinal properties.

In this study, 30 phytocomponents from rosemary, thyme, parsley, sage and basil were selected to evaluate their potential to inhibit biglycan so as to block the activation of the FAK signaling pathway and in turn, arrest metastasis in non-small cell lung cancer. Amongst these 30 phytocomponents, ten were selected according to their binding interactions with the protein for further studies with the protein complex.

According to the results obtained in the present investigation, Rosmarinic acid (RA) gave the highest docking score with biglycan and FAK hence it was selected for the molecular dynamics study. Rosmarinic acid (RA) is an abundant phenolic ester found in Rosemary (Rosmarinus officinalis), Thyme (Thymus vulgaris), Basil (Ocimum basilicum) and Sage (Salvia officinalis) which has been used in oriental medicine 30,31 . Many studies have reported that RA possesses variety of biological effects including antiinflammatory, anti-diabetic, and anti-cancer activities ${ }^{32}$. In a study by Xu et al. ${ }^{33}$, it has been reported that Rosmarinic acid inhibited bone metastasis induced by breast cancer, which was substantiated in a further study 34 .

Analysis of binding energy values for Rosmarinic acid yielded the highest docking score as compared to other phytocomponents. Molecular docking study of BGN-RA complex with FAK showed a binding interaction of -763.82 $\mathrm{KJ} / \mathrm{mol}$ which is less than the binding score of BGN-FAK complex which indicated that the phytocomponent rosmarinic acid effectively inhibits the interaction of BGN with FAK. The interaction energy of FAK-RA complex with BGN suggests the converse as the interaction energy is higher than that of the BGN-RA complex with FAK complex, which reveals that in the presence of this specific phytocomponent, its interaction and binding affinity is greater towards BGN. Consequently, it does not bind strongly with FAK. Molecular dynamics of these complexes showed the initial and subsequent changes in structure in terms of RMSD values. The stability of the BGN-RA with FAK complex shows that the phytocomponent interaction can inhibit the binding of BGN with FAK, hence activation of focal adhesion kinase will be impaired and thus metastasis can be controlled.

This study therefore provides evidence that Rosmarinic acid could block the binding interaction of BGN to FAK and consequently impair the activation of FAK signaling, which in turn, would effectively inhibit metastasis. This molecule would therefore prove to be of immense significance in controlling metastasis in Non- small cell lung cancer.

\section{CONCLUSION:}

Molecular dynamics simulations and interaction energy analysis confirmed the selectivity of Rosmarinic acid (RA) as potential inhibitor based on the conformational and dynamic differences observed between the residues in the Protein active sites. Investigating the binding interactions of BGNRA-FAK complex prove significant in inhibiting the interaction of BGN to FAK which can efficiently arrest metastasis in NSCLC.

\section{Conflict of interest statement:}

There are no competing interests to declare.

\section{REFERENCES:}

1. Mendes R, Carreira B, Baptista PV, Fernandes AR. Non-small cell lung cancer biomarkers and targeted therapy-two faces of the same coin fostered by nanotechnology. Expert Review of Precision Medicine and Drug Development. 2016; 1(2):15568.

2. American Cancer Society. Cancer Facts \& Figures 2018. Atlanta: American Cancer Society; 2018

3. Malik PS, Raina V. Lung cancer: Prevalent trends \& emerging concepts. The Indian Journal of Medical Research. 2015; 141(1):5-7.

4. Tsao AS, Scagliotti GV, Bunn Jr PA, Carbone DP, Warren GW, Bai C, De Koning HJ, Yousaf-Khan AU, McWilliams A, Tsao MS, Adusumilli PS. Scientific advances in lung cancer 2015. Journal of Thoracic Oncology. 2016; 11(5):613-38.

5. Chen H, Xiao Q, Hu Y, Chen L, Jiang K, Tang Y, Tan Y, Hu W, Wang Z, He J, Liu Y. ANGPTL1 attenuates colorectal cancer metastasis by up-regulating microRNA-138. Journal of Experimental \& Clinical Cancer Research. 2017; 36(1):78.

6. Indovina P, Marcelli E, Pentimalli F, Tanganelli P, Tarro G, Giordano A. Mass spectrometry-based proteomics: The road to lung cancer biomarker discovery. Mass spectrometry reviews. 2013; 32(2):129-42.

7. Ahn JM, Cho JY. Current serum lung cancer biomarkers. J Mol Biomark Diagn. 2013; 4:2-7.

8. Riihimäki M, Hemminki A, Fallah M, Thomsen H, Sundquist K, Sundquist J, Hemminki K. Metastatic sites and survival in lung cancer. Lung Cancer. 2014; 86(1):78-84. 
9. Hu L, Zang MD, Wang HX, Li JF, Su LP, Yan M, Li C, Yang QM, Liu BY, Zhu ZG. Biglycan stimulates VEGF expression in endothelial cells by activating the TLR signaling pathway. Molecular oncology. 2016; 10(9):1473-1484.

10. Hu L, Duan YT, Li JF, Su LP, Yan M, Zhu ZG, Liu BY, Yang QM. Biglycan enhances gastric cancer invasion by activating FAK signaling pathway. Oncotarget 2014; 5(7):1885-1896.

11. Thomsen R and Christensen M. MolDock: A New Technique for High-Accuracy Molecular Docking J. Med. Chem. 2006; 49(11):3315 - 3321

12. Krieger E and Vriend G. New ways to boost molecular dynamics simulations J.Comput.Chem. 2015; 36: 996-1007.

13. Hornak V, Abel R, Okur A, Strockbine B, Roitberg A, Simmerling C. Comparison of multiple AMBER force field and development of improved protein backbone parameters Proteins. 2006; 65:712-725.

14. Krieger E, Darden T, Nabuurs SB, Finkelstein A, Vriend G. Making optimal use of empirical energy functions: force-field parameterization in crystal space. Proteins. 2004; 57(4):67883.

15. PDBID- 2FT3 Scott PG, Dodd CM, Bergmann EM, Sheehan JK Bishop PN. Crystal structure of the biglycan dimer and evidence that dimerization is essential for folding and stability of class I small leucine-rich repeat proteoglycans. Journal of Biological Chemistry. 2006; 281(19):13324-32.

16. PDBID- 4NY0 Brami-Cherrier K, Gervasi N, Arsenieva D, Walkiewicz K, Boutterin MC, Ortega A, Leonard PG, Seantier B, Gasmi L, Bouceba T, Kadaré G. FAK dimerization controls its kinase-dependent functions at focal adhesions. The EMBO journal. 2014:e201386399.

17. Chen $\mathrm{C}$, Huang $\mathrm{H}, \mathrm{Wu} \mathrm{CH}$. Protein bioinformatics databases and resources. Protein Bioinformatics: From Protein Modifications and Networks to Proteomics. 2017:3-9.

18. Kim S, Thiessen PA, Bolton EE, Chen J, Fu G, Gindulyte A, Han L, He J, He S, Shoemaker BA, Wang J. PubChem substance and compound databases. Nucleic acids research. 2015; 44(D1):D1202-13.

19. Miyamoto S, Kollman PA. SETTLE: an analytical version of the SHAKE and RATTLE algorithm for rigid water models. J.Comp.Chem.1992; 13: 952-962.

20. Cerutti, DS, Swope WC, Rice JE, Case DA. ff14ipq: a selfconsistent force field for condensed-phase simulations of proteins. Journal of chemical theory and computation. 2014; 10(10):4515-4534.

21. Patel CN, Georrge JJ, Modi KM, Narechania MB, Patel DP, Gonzalez FJ, Pandya HA. Pharmacophore-based virtual screening of catechol-o-methyltransferase (COMT) inhibitors to combat Alzheimer's disease. Journal of Biomolecular Structure and Dynamics. 2017; 28:1-20.

22. Popper HH. Progression and metastasis of lung cancer, Cancer and Metastasis Reviews. 2016; 35(1):75-91.

23. Maishi N, Hida K. Tumor endothelial cells accelerate tumor metastasis. Cancer Science 2017; 108: 1921-1926.

24. Aronsohn MS, Brown HM, Hauptman G, Kornberg LJ. Expression of focal adhesion kinase and phosphorylated focal adhesion kinase in squamous cell carcinoma of the larynx. Laryngoscope. 2003; 113: 1944-1948.

25. Grisaru-Granovsky S, Salah Z, Maoz M, Pruss D, Beller U, BarShavit R. Differential expression of protease activated receptor 1 (Par1) and pY397FAK in benign and malignant human ovarian tissue samples. Int J Cancer. 2005; 113: 372 378.

26. Megison ML, Stewart JE, Nabers HC, Gillory LA, Beierle EA. FAK inhibition decreases cell invasion, migration and metastasis in MYCN amplified neuroblastoma. Clin Exp Metastasis. 2013; 30: 555-568.

27. Zhao J, Guan JL. Signal transduction by focal adhesion kinase in cancer. Cancer Metastasis Rev. 2009; 28: 35-49.

28. Javed, H, Erum, S, Tabassum, S, and Ameen, F. An overview on medicinal importance of thymus vulgaris. Journal of Asian Scientific Research. 2013; 3(10): 974-982.

29. Yesil-Celiktas O, Sevimli C, Bedir E, Vardar-Sukan F. Inhibitory effects of rosemary extracts, carnosic acid and rosmarinic acid on the growth of various human cancer cell lines. Plant foods for human nutrition. 2010; 65(2):158-63.

30. Zimmermann BF, Walch SG, Tinzoh LN, Stühlinger W, Lachenmeier DW. Rapid UHPLC determination of polyphenols in aqueous infusions of Salvia officinalis L. (sage tea). Journal of Chromatography B. 2011; 879(24):2459-2464.

31. Chen Y, Zhu Z, Guo Q, Zhang L, Zhang X. Variation in concentrations of major bioactive compounds in Prunella vulgaris L. related to plant parts and phenological stages. Biological Research. 2012; 45(2):171-175.

32. Amoah SK, Sandjo LP, Kratz JM, Biavatti MW. Rosmarinic acid-pharmaceutical and clinical aspects. Planta medica. 2016; 82(05):388-406.

33. $\mathrm{Xu} \mathrm{Y,} \mathrm{Jiang} \mathrm{Z}$, Ji G, Liu J. Inhibition of bone metastasis from breast carcinoma by rosmarinic acid. Planta medica. 2010 76(10):956-962.

34. Xu Y, Xu G, Liu L, Xu D, Liu J. Anti-invasion effect of rosmarinic acid via the extracellular signal-regulated kinase and oxidation-reduction pathway in Ls174-T cells. Journal of cellular biochemistry. 2010; 111(2):370-379. 